\title{
Radiant measurement accuracy of micrometeors detected by the Arecibo 430 MHz Dual-Beam Radar
}

\author{
D. Janches ${ }^{1,}$, M. C. Nolan ${ }^{1}$, and M. Sulzer ${ }^{1}$ \\ ${ }^{1}$ Arecibo Observatory, HC3 Box 53995, Arecibo, Puerto Rico 00612 \\ *Presently at CIRES, University of Colorado, 216 UCB, Boulder, CO 80309, USA
}

Received: 1 October 2003 - Published in Atmos. Chem. Phys. Discuss.: 12 January 2004

Revised: 26 March 2004 - Accepted: 28 March 2004 - Published: 15 April 2004

\begin{abstract}
Precise knowledge of the angle between the meteor vector velocity and the radar beam axis is one of the largest source of errors in the Arecibo Observatory (AO) micrometeor observations. In this paper we study $\sim 250$ high signal-to-noise ratio (SNR) meteor head-echoes obtained using the dual-beam $430 \mathrm{MHz}$ AO Radar in Puerto Rico, in order to reveal the distribution of this angle. All of these meteors have been detected first by the radar first side lobe, then by the main beam and finally seen in the side lobe again. Using geometrical arguments to calculate the meteor velocity in the plane perpendicular to the beam axis, we find that most of the meteors are travelling within $\sim 15^{\circ}$ with respect to the beam axis, in excellent agreement with previous estimates. These results suggest that meteoroids entering the atmosphere at greater angles may deposit their meteoric material at higher altitudes explaining at some level the missing mass inconsistency raised by the comparisson of meteor fluxes derived from satellite and traditional meteor radar observations. They also may be the source of the observed high altitude ions and metalic layers observed by radars and lidars respectively.
\end{abstract}

\section{Introduction}

The Arecibo Observatory (AO) $430 \mathrm{MHz}$ radar detects $\sim 10000$ events daily, and measures their radial velocity with great accuracy. We obtain this high accuracy by measuring the Doppler shift of the meteor head-echoes, which are radar reflections from a plasma travelling with the meteoroid. The largest source of error in these observations arises from the lack of precise knowledge of the position of the meteoroid as it flies through the $305 \mathrm{~m}$ diameter radar beam (at $\sim 100 \mathrm{~km}$ of altitude), and their exact angle of entry. Very

Correspondence to: D. Janches

(diego.janches@colorado.edu) precise Doppler measurments of the line-of-sight meteor velocity give estimates with less than $1 \%$ errors for radial velocities between a few $\mathrm{km} / \mathrm{s}$ and $\sim 70 \mathrm{~km} / \mathrm{s}$. We have argued in the past, based on dynamical arguments and geocentric meteor velocity measurments (Meisel et al., 2002; Janches et al., 2003), that, although unknown, the relative angle between the radar beam and the meteoroid vector velocity must be small, less than $15^{\circ}$. We will refer to this angle as the meteor off-axis angle for the rest of this paper. Knowledge of this angle is crucial for the precise radiant determination needed for orbital calculations (Janches et al., 2001; Meisel et al., 2002). A small error will also be introduced in our meteoroid size estimates (Janches et al., 2000b) and calculations of meteoric mass flux into the upper atmosphere due to these meteoroids (Mathews et al., 2001; Raizada et al., 2003). These issues will be discussed in more detail later in this paper.

Figure 1 shows velocity distributions obtained at $\mathrm{AO}$ with the dual-beam radar system during the 04:00-06:00 $\mathrm{h}$ AST (UT-4h) interval of 21 January 2002. Although our observations covered the whole night, we present and discuss only the distributions between 04:00 and 06:00 $\mathrm{h} \mathrm{AST}$, the period during which the meteor rate detected by the AO radar is maximum ( $\sim 40$ events per minute; Janches et al., 2003) and the result reported here is clearest. The high event rate is due to the fact the AO radar points towards the so-called apex, the direction of the Earth's motion in its orbit around the Sun, where particles caught by the Earth, as well as head-on collisions, are present, unlike the antapex direction where a particle must travel sufficiently fast to reach the Earth from behind in its orbit (Janches et al., 2000a). The "North" (Fig. 1a) velocity distribution was detected by the AO Gregorian feed (GF) when it was pointed $15^{\circ}$ from the zenith looking north of AO and the "South" distribution (Fig. 1c) was obtained when the antenna was pointed $15^{\circ}$ from the zenith looking south of AO. Figure 1b shows the distribution from the detections of the line feed (LF) looking vertical at AO. The 

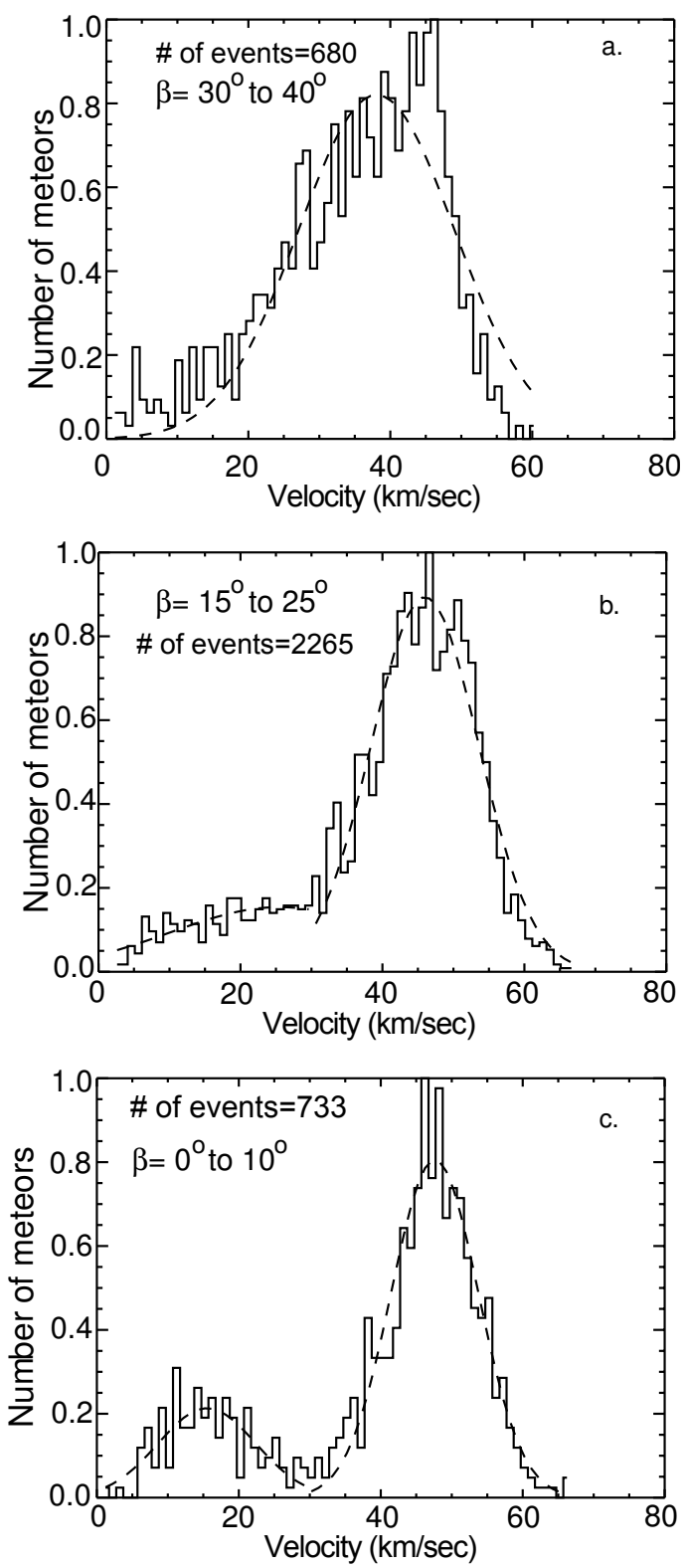

Fig. 1. Observed geocentric meteor velocity distributions on the local morning (04:00-06:00 h AST) of 22 January 2002. The upper panel displays the velocity distribution detected by the GF when pointing north of $\mathrm{AO}$, the middle displays the events detected by the LF pointing vertically and the bottom panel are the events detected by the GF when pointing south of AO. The dashed lines represent gaussian fits to the distributions and the $\beta$ values are the ecliptic latitude range covered by the $\mathrm{AO}$ beams during this interval.

complete description of these velocity distributions is presented in Janches et al. (2003). From these figures the dependence of the distribution shape with topocentric declination (which is a function of radiant ecliptic latitude) can be seen. While the "North" velocity distribution has a single peak, the "South" distribution is clearly bimodal with very few events with velocities of the order of $30 \mathrm{~km} / \mathrm{s}$. The "Ver-
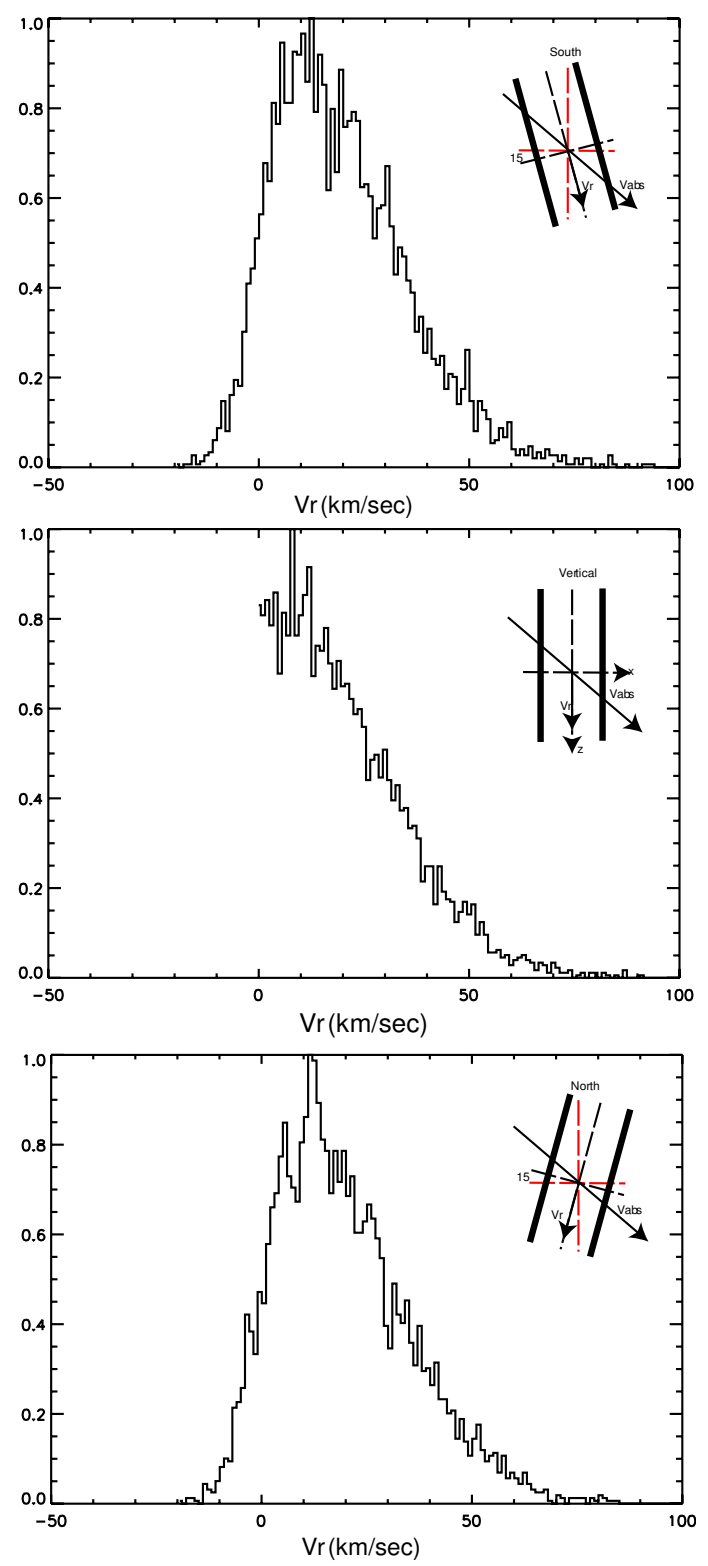

Fig. 2. Simulated line-of-sight velocities for the three pointing directions used for the radar observations presented here.

tical" distribution seems to be a mixture of the "North" and "South", where the bimodal characteristics are still present, but with some $30 \mathrm{~km} / \mathrm{s}$ objects. As pointed out in Janches et al. (2003), these differences in the distributions as well as the lack of events with velocities of the order of $30 \mathrm{~km} / \mathrm{s}$ cannot be due to an instrumental bias, because we are using the same instrument (the GF) to monitor two different areas of the sky. If the absence of meteors were due to an instrumental bias, then the effect should be present in both the "North" and "South" distributions.

In order to further explore this issue, we performed a simple simulation where uniform random distributions in meteor 
vector velocity are assumed. We then calculate the line-ofsight velocity for the different pointing directions presented in Fig. 2. Note that the "North" and "South" simulated velocity distributions are identical, while the "Vertical" distribution decreases with increasing velocity. It is deduced from this simulation that the particles that produced the meteors detected at Arecibo are not traveling in random directions and that for each pointing direction there is a prefered radiant distribution. We suggest the scenario where a filtering effect occurs in which, for a particular pointing direction, the radar detects mainly particles with vector velocities within a narrow angular range with respect to the beam axis. In the next section we describe the methodology used to determine an upper limit to this angular range. We present and discuss the results in Sect. 3 and explore the posibles causes of this effect and give the major implications on our general micrometeor results.

\section{Methodology}

For this study, we use a sample of 265 (out of $\sim 40000$ ) radar meteor head-echoes that show the largest $(<1000)$ signalto-noise ratio (SNR) detected at $\mathrm{AO}$ during the nights of 21 January and 25 June 2002. Figure 3 displays the range-timeintensity (RTI) plot of one of these events. Note that the meteor return in this figure shows three parts. The first and last part are the return detected by the radar first diffraction lobe while the middle and strongest part is the detection of the meteor while traveling through the main beam. All the meteors in the subset discussed here show this characteristic. If we assume that the meteor travels through the center of the main beam (Fig. 4) we can calculate from the duration of the event how long it took to cross the length of the main beam $(\sim 300 \mathrm{~m})$. From this calculation, we can estimate the largest tangential velocity that the meteor would have as

$V_{T}=\frac{0.3 \mathrm{~km}}{T_{M B}}$,

where $V_{T}$ is the tangential velocity and $T_{M B}$ is the duration of the meteor in the radar main beam. The estimation of the meteor off-axis angle is trivial once we know the tangential and radial component of the meteor velocity and is given by

$\alpha=\tan ^{-1}\left(\frac{V_{T}}{V_{r}}\right)$

where $V_{r}$ is the radial velocity. Note that if the meteor does not travel throught the center of the main beam, then the distance it travels is shorter hence the tangential velocity appears artificially higher and $\alpha$ is smaller too: that is why this estimate represents the upper limit for the off-axis angle.

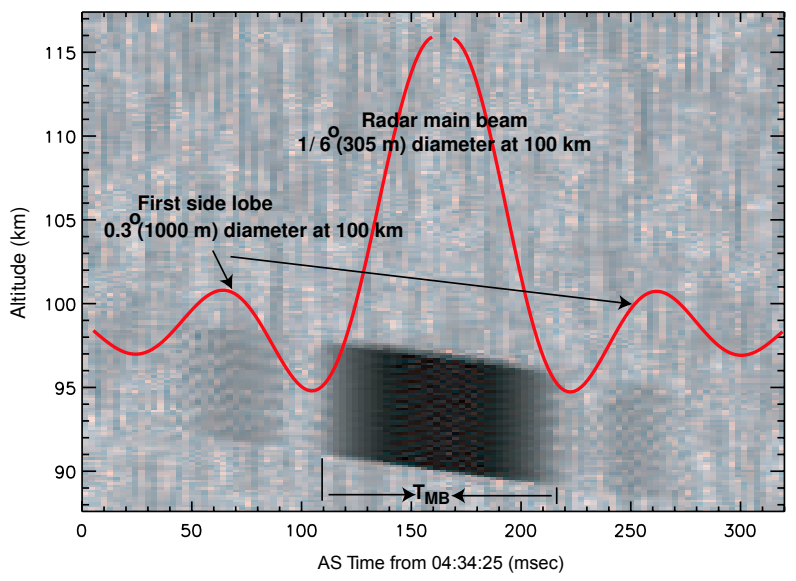

Fig. 3. Range-time-intensity (RTI) plot showing a meteor event that begins at $\sim 93 \mathrm{~km}$ and shows a range extent that corresponds to the $45 \mu$ s uncoded radar pulse. The interpulse period (IPP) is $1 \mathrm{~ms}$ and the sample rate is $1 \mu \mathrm{s}(150 \mathrm{~m})$. The red line is a representation of the radar beam pattern indicating when the meteor crosses the side lobes or the main beam.

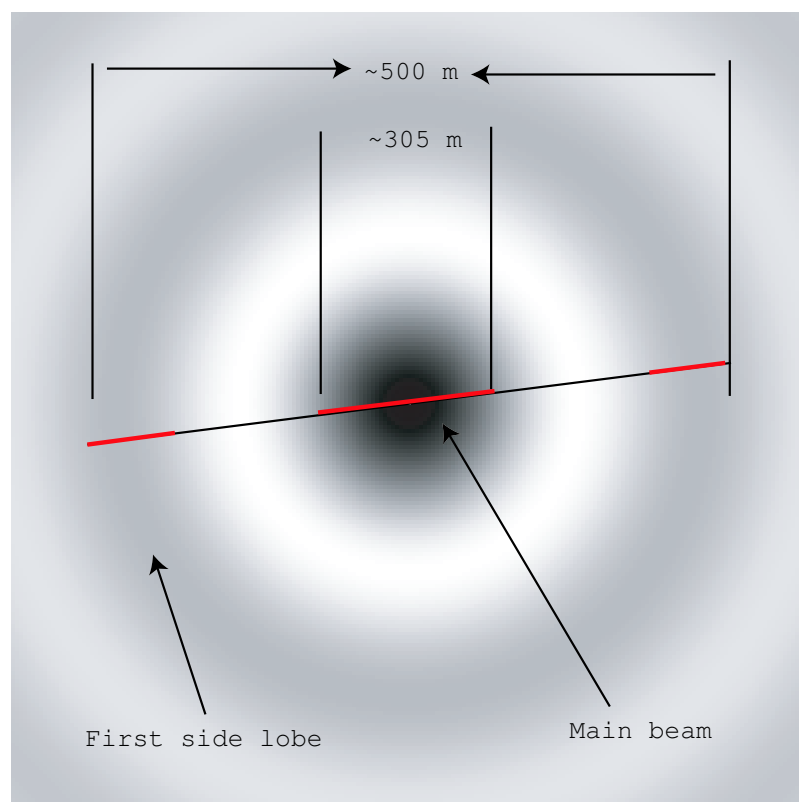

Fig. 4. A cartoon representing the radar beam seen from above and a meteor crossing the beam through the center. The red part of the meteor path represents the portions of the meteor that are detected by the radar while the black ones are the interval when no return was recorded. See Fig. 3.

\section{Discussion}

The distribution of estimated meteor off-axis angles from the methodology described in the previous section is presented in Fig. 5 (red dashed line). This histogram contains 265 events discussed in this paper. A gaussian curve is fitted to the distribution and is given by 


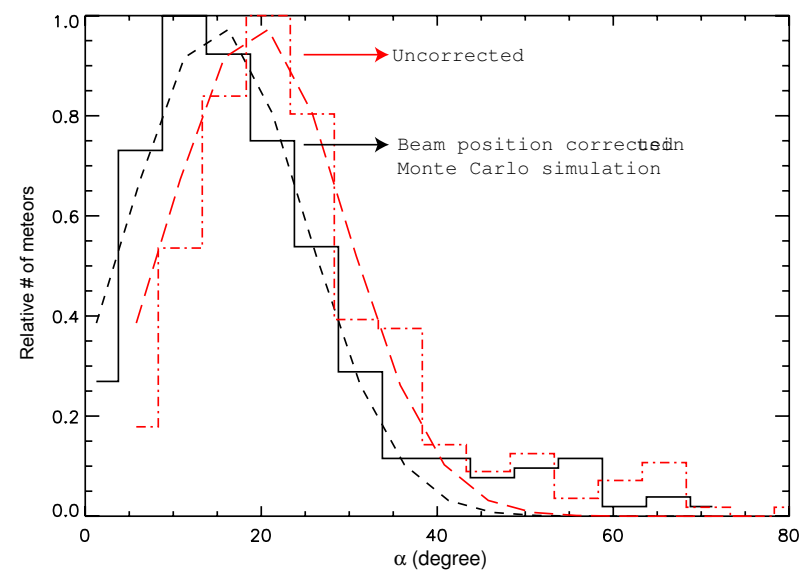

Fig. 5. Meteor off-axis distribution estimated from the geometrical study discuss in this paper.

$f(\alpha)=A_{0} \times \exp \left(-\frac{(\alpha-\bar{\alpha})^{2}}{\sigma^{2}}\right)$

where $\bar{\alpha}$ is the mean angle and $\sigma$ is the standard deviation. The fitted parameters for the curve presented in Fig. 5 are given in Table 1. In addition, Table 1 shows the fits for the data sets with same radar pointing characteristics. The results presented in Table 1 show that, independently of antenna feed or radiant observed, the detected angular distribution is identical for the three directions and both feeds, suggesting that there is a filtering process which prevents particles with large off-axis angles to reach the radar volume between 80 and $120 \mathrm{~km}$ of altitude. Figure 6 (red dashed line) displays the meteors per unit solid angle. This figure suggests that AO cannot detect meteors with small off-axis angle $\left(<7^{\circ}\right)$ which makes no physical sense. This effect is an artifact of the assumption that all the events are travelling through the radar beam center. In order to correct for this effect, we perform a further correction by randomly choosing the meteor position within the radar beam. The results of this correction are shown in both Figs. 5 and 6, as solid black lines. Gaussian curves were also fitted to these results and their parameters are presented in Table 2. We can see from Fig. 5 that for the corrected distribution the avergae off-axis angle is $15^{\circ}$. Taking the 1/e point in the gaussian curve (black solid line) in Fig. 6, which looks as expected, we can estimate that most of the events are within $15^{\circ}$ the radar beam axis.

We now explore the different causes that could produce this effect. We first note that a particle traveling at $\sim 50 \mathrm{~km} / \mathrm{s}$ at an off-axis angle of $90^{\circ}$ will spend $\sim 6 \mathrm{~ms}$ inside the $\mathrm{AO}$ radar main beam. Since the IPP used in our observations is $1 \mathrm{~ms}$, there is no reason why these events should fly undetected through the beam. In fact, we measure a small sample of meteors which show low line-of-sight velocities and short durations and possibly some of these events are fast particles crossing the beam with large tangential velocity components. However, it is difficult to distinguish be-

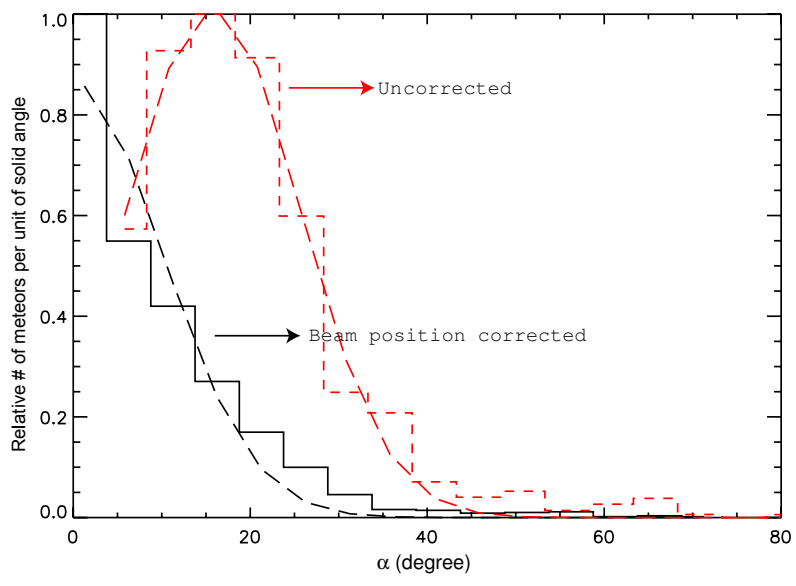

Fig. 6. Meteor per unit of solid angle as a function of the off-axis angle estimated from the geometrical study discuss in this paper.

tween meteors and space debris when the line-of-sight velocity is lower than the Earth's escape velocity $(11.2 \mathrm{~km} / \mathrm{s})$. An other posibility is that the head echo is aspect sensitive, that is the radar return is stronger for meteor traveling at low off-axis angles. However, Close et al. (2002) reported for the first time high polarization ratio measurements of meteor head-echoes utilizing ALTAIR, suggesting that the meteor head-echo is approximately spherical in shape (i.e. the radar cross section is isotropic). Theoretical results reported by Plane (2004)(Fig. 3a) show curves of height versus meteoroid velocity at which a $10 \mu \mathrm{g}$ meteoroid will reach a given temperature. For temperatures of $\sim 1500$ to $2000 \mathrm{~K}$ (stony meteoroid melting temperatures) the curves are in excellent agreement with the observed curves resulting from $\mathrm{AO}$ observations (Janches et al., 2003). The results presented here are also in excellent agreement with previous estimates using dynamical arguments (Meisel et al., 2002). This suggest that the filtering effect is produced mainly because particles with large off-axis angle would interact with sufficient atmosphere so that they ablate or stop before reaching the small radar volume centered at $\sim 100 \mathrm{~km}$ of altitude. This would imply that a portion of the incoming meteoric material is ablated and deposited at higher altitudes than the so called meteor ablation zone. Estimation of the meteoric mass detected by the AO radar gives $\sim 2000$ tons per year over the whole Earth for particles in the $0.5-100 \mu \mathrm{m}$ size range (Mathews et al., 2001; Raizada et al., 2003). This estimate raised some controversies since it is lower than a widely used estimate given by Love and Brownlee (1993) but in better agreement, although still lower than other estimates (Ceplecha et al., 1998; Rietmeijer, 2000). For the same particle mass range detected at $\mathrm{AO}\left(10^{-5}-10 \mu \mathrm{g}\right)$, these estimates were mainly derived from satellite measurements which are much higher $(\sim 500 \mathrm{~km})$ than the altitudes at which radar meteor activity is maxima $(\sim 105 \mathrm{~km})$. Hughes (1978) showed that there is a large discrepancy between the meteor fluxes derived from 
satellite and conventional meteor radars observations. If a portion of the meteor influx is deposited at high altitudes as these results suggest, then it could explain at least at some level the missing mass discrepancy raised by the comparisson between conventional meteor radars and satellite measurements. In addition, Tepley et al. (2003) report lidar observations of $\mathrm{Ca}^{+}$layers at altitudes near $120 \mathrm{~km}$ which are correlated to Ne layers observed with the AO radar in ISR mode, and appear to be descending from above this zone. Höffner and Friedman (2003) report lidar measurements at high altitude $(>110 \mathrm{~km}) \mathrm{K}, \mathrm{Ca}, \mathrm{Ca}^{+}, \mathrm{Fe}$ and Na layers. The authors note that despite the different seasonal characteristics between the different low altitude metalic layers, these high altitude ones show similarity along the year and different locations and they are likely formed from direct meteoric deposition. Given all these facts, we are led to conclude that micon-size particles which enter the atmosphere at large angles will ablate at higher altitudes and could be regarded to some degree as the source for the ions which produce the descending layers and high altitude metalic layers and accounting for the lower total mass flux measured by radars compared with satellite observations.

Chau and Woodman (2004) using observations performed with the $50 \mathrm{MHz}$ Jicamarca (JRO) radar in interferometric mode showed that the detected particles have radiants within a narrow angular range around the apex direction. Sulzer (2004), using regular AO incoherent scatter radar of the ionosphere, show that AO and JRO seem to observe the same flux and that this "prefered" directionality on the meteor radiant distribution helps also explain to some degree the differences oberved in the high velocity component of the meteor geocentric velocity distributions resulted from AO observations (Fig. 1; Janches et al., 2003) in the sense that the "South" distribution results from observing close to the ecliptic plane right into the meteor flow, while the "North" distribution is the same flow but shifted by a cosine factor (Sulzer, 2004). The slow component seem to be independent of this effect. The strong diurnal behavior of the meteor rate observed at AO Janches et al. (2003) suggests that the difference in the velocity distributions are more likely from a combination of both effects argue here, atmospheric filttering and geometrical. If only the geometrical effect would be present, $\mathrm{AO}$ should detect particles as soon as the radiant is above the horizon and this is not the case. There is practically no meteor activity until after $\sim$ 02:00-03:00 AST (Janches et al., 2003). Not only AO detects a micrometeors resulting from particles travelling within a narrow angular spread with respect to the radar beam axis but also the flux is non isotropic. This implies that, due to the pointing characteristics of the AO system during the observations presented here, the "South" distribution represents absolute velocities since it is pointing directly into the radiant of the meteor flow. This suggests then that the bi-modal characteristics of the meteor velocity distribution is a real feature of the meteor population observed by these radars indicating the presence of two popu-
Table 1. Gaussian fitting parameters.

\begin{tabular}{llccr}
\hline Antenna Feed & Pointing & $\mathrm{A}_{0}$ & $\bar{\alpha}$ (degrees) & $\sigma$ (degrees) \\
\hline Line & Vertical & 0.82 & 22.4 & 8.5 \\
Gregorian & North & 0.72 & 22.6 & 9.0 \\
Gregorian & South & 0.99 & 20.8 & 1.7 \\
All & $\mathrm{t}$ & 0.86 & 21.7 & 9.3 \\
All (corrected) & $\mathrm{t}$ & 0.98 & 15.0 & 10.0 \\
\hline
\end{tabular}

Table 2. Gaussian fitting parameters for Fig. 6.

\begin{tabular}{lclc}
\hline Beam Position Correction & $\mathrm{A}_{0}$ & $\bar{\alpha}$ (degrees) & $\sigma$ (degrees) \\
\hline Yes & 0.86 & $1.9 \times 10^{-6}$ & 10.1 \\
No & 1.01 & 15.83 & 9.74 \\
\hline
\end{tabular}

lations of particles in the $\mathrm{AO}$ detections: a slow and less dominant with asteroidal orbital characteristics and a faster and more dominant, which orbits are mainly retrograde (Janches et al., 2001). The fast distribution can either be the results of particles of cometary origin or small charged particles that have been accelerated by electromagnetic effects with the interplanetary magnetic field (Morfill and Grün, 1979; Janches et al., 2001). The slower population are probably a mix of asteroidal debris, space debris and/or fast particles with large tangential velocities with respect to the $\mathrm{AO}$ radar beam axis. In any case, the fundamental result of this paper is that for a particular pointing direction, events are travelling within a narrow angular distribution with respect to the beam axis, and that the lack of knowledge of the precise path of the particles trhough the very narrow AO $430 \mathrm{MHz}$ beam does not introduce significant error to our dynamical mass estimates or our orbital results (Janches et al., 2001; Meisel et al., 2002).

\section{Conclusions}

In this paper we presented $\sim 250$ high signal-to-noise (SNR) ratio meteor head-echoes observations obtained using the dual-beam $430 \mathrm{MHz}$ Arecibo Observatory (AO) Radar in Puerto Rico. Using the known diffraction pattern of the telescope, we applied a geometrical calculation in order to estimate the distribution of the meteor off-axis angle (i.e. angle between the vector velocity and the radar beam axis). We find that meteors mostly travel with radiants within $\sim 15^{\circ}$ with respect to the radar beam axis, in excellent agreement with previous estimates. This uncertainty does not introduce significant error to our general results of meteoric mass estimates and orbital characteristics of the dust. 
Acknowledgements. The Arecibo Observatory is part of the National Astronomy and Ionosphere Center, which is operated by Cornell University under cooperative agreement with the National Science Foundation. This work has been supported under NSF/CEDAR Post Doctoral program awarded to D. Janches (ATM-0004284). D. Janches wishes to thank S. Basu and R. Robinson at NSF and P. Bellaire and E. Murad at AFRL for the support that made the Radar Meteor Workshop at Arecibo possible, and all the participants for making it a very successful conference.

Edited by: J. Plane

\section{References}

Ceplecha, Z., Borovička, J., Elford, W. G., Revelle, D. O., Hawkes, R. L., V. Porubčan, and Šimek, M.: Meteor Phenomena and Bodies, Space Sci. Rev., 84, 327-471, 1998.

Chau, J. L. and Woodman, R. F.: Observations of meteor-head echoes using the Jicamarca $50 \mathrm{MHz}$ radar in interferometer mode, Atmos. Chem. Phys., 4, 511-521, 2004.

Close, S., Oppenheim, M., Hunt, S. M., and Dyrud, L.: Scattering characteristics of high-resolution meteor head echoes detected at multiple frequencies, J. Geophys. Res., 107, (A10), 1295, doi:10.1029/2002JA009253, 2002.

Höffner, J. and Friedman, J. S.: Metal layers at high altitudes: A possible connection to meteoroids, Atmos. Chem. Phys., 4, 399417, 2004

Hughes, D. W.: Meteors, Cosmic Dust, edited by McDonnell, J. A. M., Chichester, John Willey, 123-186, 1978.

Janches, D., Mathews, J. D., Meisel, D. D., Getman, V. S., and Zhou, Q. H.: Doppler studies of near-antapex UHF radar micrometeors, Icarus, 143, 347-353, 2000a.

Janches, D., Mathews, J. D., Meisel, D. D., and Zhou, Q. H.: Micrometeor observations Using the Arecibo $430 \mathrm{MHz}$ radar: I. Determination of the Ballistic Parameter from measured Doppler velocity and deceleration results, Icarus, 145, 53-63, 2000b.
Janches, D., Meisel, D. D., and Mathews, J. D.: Orbital Properties of the Arecibo Micrometeors at Earth Interception, Icarus, 150, 206-218, 2001.

Janches, D., Nolan, M. C., Meisel, D. D., Mathews, J. D., Zhou, Q. H., and Moser, D. E.: On the geocentric micrometeor velocity distribution, J. Geophys. Res., 108, (A6), 1222, 2003.

Love, S. G. and Brownlee, D. E.: A direct measurement of the terrestrial mass accretion rate of cosmic dust, Science, 262, 550553, 1993.

Mathews, J. D., Janches, D., Meisel, D. D., and Zhou, Q. H.: The Micrometeoroid Mass Flux into the Upper Atmosphere: Arecibo Results and a Comparison with Prior Estimates, Geophys. Res. Lett., 28, 10, 1929, 2001.

Meisel, D. D., Janches, D., and Mathews, J. D.: Extrasolar Micrometeors Radiating from the Vicinity of the Local Interstellar Bubble, Ap. J., 567, 323-341, 2002.

Morfill, G. E. and Grün, E.: The motion of charged dust particles in interplanetary space: I. The zodiacal dust cloud, Planet. Space Sci., 27, 1269-1282, 1979.

Plane, J. M. C.: A new time-resolved model for the mesospheric Na layer: constraints on the meteor input function, Atmos. Chem. Phys., accepted, 2004.

Raizada, S., Tepley, C. A., Janches, D., Friedman, J. S., Zhou, Q. H., and Mathews, J. D.: Lidar observations of Ca and K metallic layers at Arecibo and comparison with micrometeor sporadic activity, J. Atmos. Solar Terr. Phys., in press, 2004.

Rietmeijer, F. J. M.: Interrelationships among meteoric metals, meteors, interplanetary dust, micrometeorites and meteorites, Meteoritics Planet. Sci., 35, 1025-1041, 2000.

Sulzer, M. P.: Meteor Science from Regular Incoherent Scatter Radar Ionospheric Observations at Arecibo, Atmos. Chem. Phys., 4, 805-831, 2004.

Tepley, C. A., Raizada, S., Zhou, Q., and Friedman, J. S.: First simultaneous observations of $\mathrm{Ca}^{+}, \mathrm{K}$, and electron density using lidar and incoherent scatter radar at Arecibo, Geophys. Ress. Lett., 30, (1), 1009, doi:10.1029/2002GL01597, 2003.

Whipple, F. L.: The theory of micro-meteorites: Part II, In heterothermal atmospheres, Proc. N. A. S., 37, 19-30, 1951. 